\title{
Semiclassical Theory of High Energy Collisions and RHIC Puzzles
}

\author{
E.V.Shuryak* ${ }^{\dagger}$ \\ Department of Physics and Astronomy, \\ State University of New York, Stony Brook NY 11794-3800
}

\begin{abstract}
The semiclassical theory of tunneling through the topological barrier in gauge theories was until recently focused only on virtual processes in the vacuum, described by the zero-energy Euclidean solutions - the instantons. Its applications for high energy collisions in a QCD context are relatively recent. Like in quantum mechanics, the semiclassical path naturally splits into a sub-barrier Euclidean part, followed by a non-trivial over-the-barrier Minkowski evolution. These two are separated by the familiar turning states, in a form of purely magnetic clusters. Recent technical developments included simple analytic solution of the Yang-Mills equations for the turning states, analytic and numerical solutions for their explosions (in Minkowski time), and recently an analytic solution of the Dirac equation in the field of exploding cluster, describing production of $2 N_{f}$ light quarks. There is also progress in calculation of the cross section itself. The phenomenological part of the talk includes brief discussion of possible cluster formation in hadronic $\left(\mathrm{pp}, \gamma^{*} p\right)$ reactions, as a part of "soft Pomeron". We also briefly review puzzling results of the first RHIC runs, and speculate to which extent high rate of quark pair production and other features of topological clusters may help to explain those puzzles. Especially important is recently pointed out observation that coherence of classical glue and the specific geometry of the exploding clusters may significantly enhance the so called jet quenching.
\end{abstract}

\section{QUANTUM MECHANICS OF THE GLUE AND THE HIGH EN- ERGY COLLISIONS}

\subsection{Introduction}

In very general terms, high energy collisions allow virtual fields -part of the wave function of the target or projectile - to become real. In QED the simplest example of that is

${ }^{*}$ Speaker.

${ }^{\dagger}$ Supported in part by the US DOE grant. 
Weizsacker-Williams approximation, describing how boosted virtual Coulomb field may become real photons. In pQCD the so called Lipatov vertex play the same role, describing how virtual gluon becomes real (on-shell) ones: it is the basis of celebrated BFKL hard Pomeron.

Similar phenomena takes place non-perturbatively. The virtual fields in hadrons and in the vacuum itself, if promptly excited by a collisions, produce certain real objects. Sudden excitation of the part of vacuum wave function under the barrier also produces certain real objects $^{1}$ : those are states on the barrier. Surprisingly, only recently they have been studied in QCD [1], $2 \overline{2}$.

So, promptly excited glue is not really several gluons: it should appear first as gluomagnetic topological clusters of well defined structure. The main idea is very simple: there is no time to change topology. It, by itself, originates from two major sources: studies of the non-perturbative tunneling phenomena in the QCD vacuum [3i] a decade ago in the discussion of high energy collisions in electroweak theory [i $\left[\begin{array}{l}\overline{4} \\ {[}\end{array}\right]$. Recent

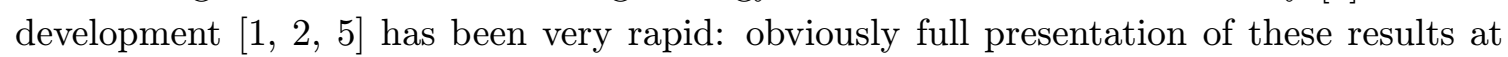
technical level is done elsewhere and here I only try to present an emerging physical picture. More details and applications to heavy ion collisions (event-by-event fluctuations and $J / \psi$ suppression) can be found in my preprint [i్6]

\subsection{Topological coordinates, tunneling paths and the turning states}

Schematically YM quantized field can be viewed as many coupled non-linear oscillators with simple potential of the type $\vec{B}^{2} \sim O\left(A^{2}\right)+O\left(A^{3}\right)+O\left(A^{4}\right)$, with coordinates being the potentials $A_{m}^{a}, \mathrm{a}=1-3$ (since we will limit ourselves to $\left.\mathrm{SU}(2)\right)$ and $\mathrm{m}=1-3$. In the $A_{0}=0$ gauge $\frac{d \vec{A}}{d t}=\vec{E}$ and the electric part of the energy is identified as the kinetic term.

One combination of fields, the Chern-Simons number, have certain topological properties

$$
N_{C S}=\int d^{3} x K_{0}, \quad K_{\mu}=-\frac{1}{32 \pi^{2}} \epsilon^{\mu \nu \rho \sigma}\left(\mathcal{G}_{\nu \rho}^{a} \mathcal{A}_{\sigma}^{a}-\frac{g}{3} \epsilon^{a b c} \mathcal{A}_{\nu}^{a} \mathcal{A}_{\rho}^{b} \mathcal{A}_{\sigma}^{c}\right)
$$

The potential energy of Yang-Mills field versus this topological coordinate $N_{C S}$ is schematically shown in Fig ${ }_{1}^{1} \bar{i}$ It is periodic, with zeros at all integer points: those are "classical vacua", with zero field strengths but non-zero (and topologically distinct) $A_{m}^{a}$.

In vacuum the tunneling paths - instantons - start at one minimum of the potential and end up in another, with zero energy. In high energy collisions a sudden localization of all quantum coordinates happens, including the topological one. The system suddenly finds itself at or above the barrier ( see the dashed line (a) in Fig., (1.1). Another possibility ( shown by the dashed line (b) in Fig. . $_{1}^{\prime}$ ) is that a system at the collision moment is not under barrier, but becomes able to tunnel through it after it gets excited enough.

Whichever way the system is driven, it emerges out of the barrier via what we call " $a$ turning state", a relative of the sphaleron ${ }^{2}$ solution of electroweak theory. This is a point

\footnotetext{
${ }^{1}$ They are real in the same sense as gluons: namely at times shorter than those at which confinement sets in.

2 "Ready to fall" in Greek, according to Klinkhammer and Manton.
} 
where the path crosses the barrier and the total energy is equal to the potential one. Here the potential energy is equal to the total one, with zero momentum (the electric field). This is why those objects are born into our world as pure gluomagnetic clusters.

From there starts the real time motion outside the barrier (shown by horizontal dotted lines): here the action is real and $\left|e^{i S}\right|=1$. That means that whatever happens at this Minkowski stage has the probability 1 and cannot affect the total cross section of the process: this part is only needed for predicting the properties of the final state.

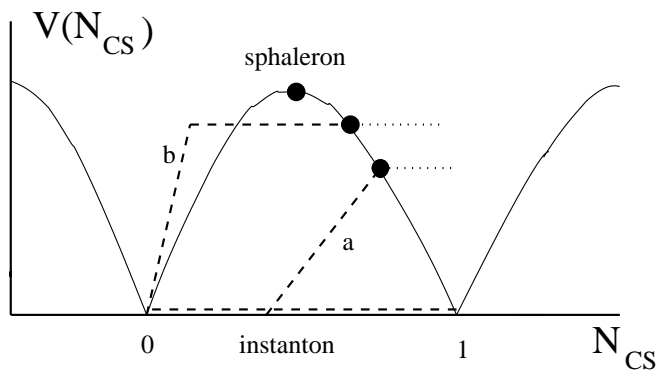

Figure 1: Potential energy of Yang-Mills field versus the Chern-Simons number $N_{c s}$.

\subsection{Turning states as a conditional minimum}

The shape of the potential and the corresponding turning states can be alternatively obtained [i.1] [i. from the minimization of the potential energy of a static Yang-Mills fields, consistent with two appropriate constraints: (i) fixed value of (corrected) Chern-Simons number $N_{C S}\left({ }_{1}, \bar{I}_{1}\right)$. (ii) fixed value of the r.m.s. size $R$. To find those one should search for the minimum of the following functional

$$
E_{\text {eff }}=\frac{1}{2} \int B_{m}^{2} d^{3} x+R\left(A_{\mu}\right) / \rho^{2}+\kappa N_{C S}\left(A_{\mu}\right) R\left(A_{\mu}\right)=\int x^{2} B_{m}^{2} d^{3} x / \int B_{m}^{2} d^{3} x
$$

where $1 / \rho^{2}, \kappa$ are two Lagrange multipliers. Although these two terms append the YM equations and make them more complicated, an analytical solution is found. Skipping the details, let me only say that the energy and (corrected) Chern-Simons number are

$$
E_{\text {stat }}=3 \pi^{2}\left(1-\kappa^{2}\right)^{2} /\left(g^{2} \rho\right), \quad \tilde{N}_{C S}=\operatorname{sign}(\kappa)(1-|\kappa|)^{2}(2+|\kappa|) / 4
$$

Eliminating $\kappa$, we find the shape of the potential plotted above. The maximum - YM sphaleron - corresponds to $\kappa=0$, and its energy is $3 \pi^{2} /\left(g^{2} \rho\right)$. If the size is determined by the mean radii of vacuum instantons $\rho=1 / 3 \mathrm{fm}[i]$ $\mathrm{GeV}$. One can view it as a magnetic ball with field lines for gluons with 1-3 colors, rotating around $\mathrm{x}, \mathrm{y}$ and $\mathrm{z}$ axes, respectively, while all gluon fields with colors 4-8 are absent.

\subsection{Explosive behavior of the turning states}

Solution of the classical YM equations describes what happens to these turning states after they are produced ${ }^{4}$ In QCD there are no Higgs scalar and its non-zero VEVs, so

\footnotetext{
${ }^{3}$ In electroweak theory this mass is about $10 \mathrm{TeV}$, so it is made of about 100 gauge bosons.

${ }^{4}$ Similar study has been made a decade ago in electroweak theory for the sphaleron, where it has been found that it decays in about $51 \mathrm{~W}, \mathrm{Z}, \mathrm{H}$ : half of the energy thus goes to acceleration.
} 
gluons are massless. This makes the process even more explosive because all harmonics with different momenta move together, with the speed of light. As described in [i] problem was solved both numerically and analytically. At large times the spherical shell has the following energy density profile

$$
4 \pi r^{2} e(r, t)=\frac{8 \pi}{g^{2} \rho^{2}}\left(1-\kappa^{2}\right)^{2}\left(\frac{\rho^{2}}{\rho^{2}+(r-t)^{2}}\right)^{3}
$$

Of course, at large times the fields becomes weak and can be decomposed into gluons: the Fourier transform of the fields provides the energy distribution of the resulting gluons. One gets about 3 gluons out of it, if the size is $1 / 3 \mathrm{fm}$. Furthermore, as shown by explicit solution of the Dirac equation [isin], a pair of each light quark flavor is taken from vacuum and accelerated to the physical energies.

The way the explosive solutions of YM and Dirac eqns is found [i5in] closely follows what we learned in complex variable courses: we start with some $\mathrm{O}(4)$-symmetric solutions and then make conformal off-center inversion, which brings solutions depending separately on $\mathrm{t}$ and $\mathrm{r}$.

\section{TOPOLOGICAL CLUSTERS IN HADRONIC COLLISIONS}

\subsection{Pomeron from instantons}

A number of authors [i, $[\overline{9}]$ have suggested that the specific behavior of the pp or the $\gamma p$ cross sections, $\sigma \sim s^{0.08}$ (the so called soft pomeron) may be related with tunneling. More specifically, a small index 0.08 is in this case proportional to the cross section of the production of topological clusters. This power, as well as the Pomeron size $\alpha^{\prime}=1 /(2 \mathrm{GeV})^{2}$ have been evaluated and gave reasonable numbers. An interesting byproduct found was the absence of odderon in this theory, which is due to the $\mathrm{SU}(2)$ color nature of fields. As discussed at this meeting, it is in agreement with all the data including recent HERA results, but in disagreement with many models.

Semiclassical soft Pomeron can be viewed as a ladder-type diagram similar to perturbative BFKL one. The difference is that the Lipatov vertex -2 virtual gluons into "physical" gluon - is substituted by a new vertex with a topological cluster produced instead of a gluon. As we argued above, it is the most natural excitation from under the barrier.

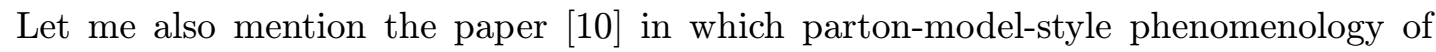
various hadronic collisions is developed. The main idea was to identify two components of the hh collisions, the color exchanges and the "color objects production", and deduce the corresponding cross sections at the partonic level. We looked at high energy $N N, \pi N, \gamma N$, and $\gamma \gamma$ cross sections which all increase with energy logarithmically for $\sqrt{s} \sim 100 \mathrm{GeV}$

$$
\sigma_{h h^{\prime}}(s)=\sigma_{h h^{\prime}}\left(s_{0}\right)+X_{h h^{\prime}} \ln \left(s / s_{0}\right)
$$

We identified the two components mentioned above with these two terms, respectively, and concentrated on the last (growing) terms. We studied whether some universal semi-hard parton-parton collisions can explain all known $X_{h h^{\prime}}$. Using fitted structure functions of $N, \pi, \gamma$ and simple scaling - each gluon can be counted as 2 quarks ${ }^{5}$ - we have expressed

\footnotetext{
${ }^{5}$ Corresponding to $\mathrm{SU}(2)$ Casimir scaling, appropriate for instanton-induced reactions.
} 
all of those with only one parameter, the value of the $q q$ cross section. With the fitted value $^{6} \sigma_{q q}=1.69 \times 10^{-3} \mathrm{fm}^{2}$ we got correct rising part of cross sections for 4 hadronic reactions. In [i] component by the first.

Finally, let me say that our approach is closely related with the instanton-induced effects in deep-inelastic scattering [il in in]. Let make only two brief comment about their approach: I agree on the evaluation of the cross section but suggest that the features of the final state cannot be understood properly without inclusion of "explosive" Minkowski stage: it reduces a number of gluons and increases their energy. The second comment is that the role of quarks, their flavor/chiral structure in this process was not so far been really incorporated.

\subsection{Direct observation of clusters in pp?}

Let us now return from the deep-inelastic to ordinary hadronic collisions. Because in those there is no large scale $Q^{2}$ available, the clusters produced have the mass and size which is determined by the typical size of the instantons in the QCD vacuum. This leads to $M \sim 3 \mathrm{GeV}, \rho \sim 1 / 3 \mathrm{fm}$, as was mentioned above. What can be their patterns of hadronization be? A significant amount of clustering in pp collisions is known for a very long time. Unfortunately, these clusters have not been studied and identified long ago. They may be related with clusters we argue about.

There are however additional complications. When a topological cluster is produced in hadronic collisions, as a colored object it is still connected by the QCD strings to some receding partons, and thus can only appear on top of some debris of the usual string fragmentation. It can be in principle be located from correlation measurements, but it is difficult to do.

One may therefore think about the so called double-diffractive (or Pomeron-Pomeron) processes in which colorless clusters are produced. Theoretically, the same diluteness parameter explains relative smallness of 3- and 4-Pomeron vertices known phenomenologically since 1970's. Experimentally let me mention one recent paper including such analysis of old data [1] ${ }_{2}$ in] in which one indeed see that such collisions indeed result in clusters of few $\mathrm{GeV}$ mass. Also intriguing is the fact that clusters with mass up to $5 \mathrm{GeV}$ decay isotropically in their rest frame. Unfortunately, the UA2 detector used was just a simple calorimeter, and we do not know anything about the structure of these clusters. RHIC detectors and especially STAR can do a lot in the pp mode, to clarify these long-standing problems.

Are there specific hadronic final states into which the outgoing quarks and gluons from the topological clusters should be projected? One may think those are glueballs, the scalar and pseudoscalar one: the best modes to look for those are $K+K-$ and $K_{s} K^{ \pm} \pi^{\mp}$, respectively. The observed decrease of HBT correlation strength with rising multiplicity in pp collisions [1] ${ }_{1}^{1}$ ] can be related with large fraction of $\eta^{\prime}, \eta, K_{s}$ long-lived sources of pions, associated with instanton-induced production.

\footnotetext{
${ }^{6}$ Note that simple parametric estimate for this cross section, namely $\pi \rho^{2} n_{\text {inst }} \rho^{4}$ gives the right magnitude.
} 


\section{RHIC PUZZLES AND THE SEMICLASSICAL THEORY}

\subsection{The main puzzles}

Before RHIC data the following competing views on early stage dynamics were discussed (i) QCD strings (RQMD,URQMD); (ii) mini-jet models (HIJING); (iii) rapid QGP equilibration (hydro and statistical models). Both (i) and (ii) predicted for RHIC smaller elliptic flow compared to SPS/AGS, while (iii) predicted it to be increased by about factor 2, due to stronger QGP push. The very first RHIC data from STAR collaboration had confirmed the explosive scenario (iii) and killed two others.

In fact both radial and elliptic collective flows are very well reproduced by ideal hydrodynamics [i] 1 in] , with the equation of state close to that derived from the lattice. Somewhat unexpectedly, it also describes well the tails of particle spectra at $p_{t} \sim 2 \mathrm{GeV}$, including the crossing of proton and pion spectra in this region. In a different words, the viscosity of matter was evaluated from deviations from hydro, and it turns out very small.

This implies that QGP is produced promptly enough, at time $\sim 1 / 2 \mathrm{fm} / \mathrm{c}$. How that happens is the first RHIC puzzle.

The second RHIC puzzle is that of very strong jet quenching, combined with large azimuthal asymmetry and large baryon fraction of particles at large $p_{t}$. The expected (and measured in pp or peripheral AA) spectrum of hard jets from parton processes is suppressed in central AA collisions by a significant factor. The measured values of azimuthal asymmetry v2 at large $p_{t}>2 \mathrm{GeV}$ exceeds even the strong quenching geometrical limit [1] backward compensating jet is only seen in pp and peripheral $\mathrm{AuAu}$, but not in more central bins. This rather directly confirms that the almond is black and strong jet quenching is a reality.

\subsection{Theory Overview}

So, why is equilibration so rapid, and nuclei are so black even for high- $p_{t}$ jets?

Re-scattering of gluons with momenta about 1-2 GeV (later called "mini-jets") have

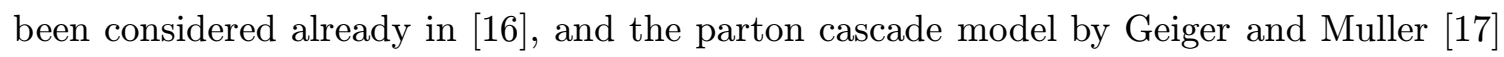
have added "branching" of virtual partons, or bremsstrahlung. Recent development [1] included Landau-Pomeranchuck-Migdal effect and other refinements: although perturbative equilibration was still found possible, its rate is suppressed by calculable powers of small coupling constant. Large potential contribution of pQCD processes 2-to-n has been discussed in [1] $19 \overline{1}$ ]: but summing of all diagrams does not seem possible and the results seem to be simply growing ${ }^{7}$ with $\mathrm{n}$. Now we definitely know that extrapolation of pQCD to momenta $Q \sim 1 \mathrm{GeV}$ fails miserably ${ }^{8}$. Probably it has to be so, because both at $Q \gg 1 \mathrm{GeV}$ (pQCD) and $Q \ll 1 G e V$ (pion Lagrangians) strong interaction is weak, and we also know it

\footnotetext{
${ }^{7}$ This would not be the case in the semiclassical theory, where $\mathrm{n}$ is always of the order of the action of the classical paths used.

${ }^{8} \mathrm{In}[20]$ it is nicely quantified by how much pQCD-based scenario with $1-2 \mathrm{GeV}$ cutoff misses what is needed: in order to reproduce elliptic flow by a parton cascade the product of the gluon density times the cross section should be increased by a factor of about 80 relative to HIJING.
} 
must be strong somewhere, to make all this non-perturbative phenomena like confinement and chiral symmetry breaking.

A completely new direction is now being developed using AdS/CFT correspondence, which potentially provides a window into QGP in a strong coupling limit. Let me mention only one paper of the kind [2 $\left[\begin{array}{l}2 \\ 1\end{array}\right]$, focused on viscosity at high $\mathrm{T}$. It was found there that although in weak coupling the relaxation time $\tau \sim 1 /\left(g^{2} N\right)^{2} \log \left(1 / g^{2} N\right) T$, in strong coupling it is simply $\sim 1 / T$. If so, hydrodynamics becomes justified, but not the parton cascades.

Although HERA data on deep inelastic scattering can still be described by the DGLAP evolution, it is not the only possible explanation: the saturation-based models have created very good fits to data and vast theoretical literature. It has been argued [22] that random classical glue, or Color Glass Condensate (CGC) can be a better description at sufficiently small $\mathrm{x}$.

In this language the point we would like to make now is that a significant part of this classical glue may be non-random but highly structured, with intrinsic topology related to tunneling. A big difference between these kinds of classical glue is due to $\vec{E} \vec{B}$ combination so important for anomaly and quark pair production: the random glue practically does not have it while the topological clusters creates ${ }^{9} \bar{u} u \bar{d} d \bar{s} s\left[\overline{\hat{p}_{1}}\right]$. If the random glue has the multiplication coefficient (ratio of final-to-initial quanta) of around 0.5 (as shown in [20 3 i] and also in our sphaleron explosion solution in pure YM), in QCD with light quarks the topological leads to about 3 quarks plus 6 fermions, with the multiplication coefficient of about 3 . It helps to solve the entropy production puzzle.

\section{Topological clusters in heavy ion collisions}

\subsection{Why heavy ion collisions are more promising than pp?}

In the QCD vacuum the non-perturbative effects operate at "semi-hard" or "substructure scale" $Q^{2} \sim 1-2 \mathrm{GeV}^{2}$, which is both the lower boundary of pQCD as well as the upper boundary of low energy effective approaches (like Nambu-Jona-Lasinio or Chiral Lagrangians). Quite different pQCD cutoff is however expected for heavy ion collisions. As argued over the years (see e.g. [1] $\left.{ }^{1} \bar{G}_{1}\right]$ ) the final state is in a Quark-Gluon Plasma phase of QCD. It is qualitatively different from the QCD vacuum: there is no confinement or chiral symmetry breaking. Therefore in QGP the cut-off is determined by a plasma-like screening, e.g. a gluon effective mass is [1] $\left.{ }_{1}^{1} \overline{6}\right] M_{g}^{2}=\frac{g^{2} T^{2}}{2}\left(\frac{N_{c}}{3}+\frac{N_{f}}{6}\right)$. Although it grows with $\mathrm{T}$, in the window $T=(1-3) T_{c}$ it is actually smaller than the pQCD cutoff in vacuum. Lattice thermodynamics data support it, and fitted quasi-particle masses (see e .g. [24in]) are $M_{T}^{g} \approx .4 \mathrm{GeV}, M_{T}^{q} \approx .3 \mathrm{GeV}$ The schematic picture of scale development with time is shown in Figure 2 . Such non-monotonous behavior of the pQCD cutoff implies that we can describe gluons (quarks) originating from exploding non-perturbative objects by classical Yang-Mills (Dirac) eqs with better confidence, provided those go into QGP in this window of parameters.

\footnotetext{
${ }^{9}$ In a way, those are simply materialized quarks from the vacuum chiral condensate.
} 


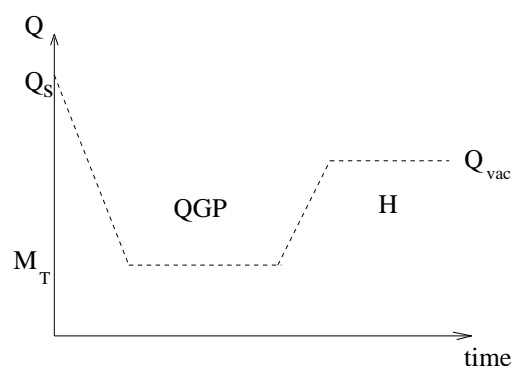

Figure 2: Schematic cut-off variation during time evolution of the system.

\subsection{How many topological clusters are there in Heavy Ion Collisions ?}

To find the semiclassical description for this process was known in 1990's as the so called holy grail problem. Three methods toward its solution have been proposed:

(i) Unitarization of the multi-gluon amplitude when it becomes strong was first sug-

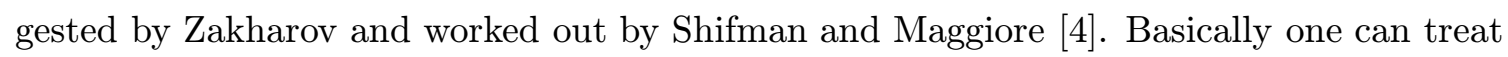
a sphaleron as a resonance.

(ii)Landau method with singular instantons was applied by Diakonov and Petrov [i] (following some earlier works which are cited there) who were able to find the opposite limit of high energies. It follows from the comparison of the two limits, that the peak is indeed very close to the sphaleron mass, and the cross section is very close to be first order in instanton diluteness.

(iii) Classical solution on the complex time plane [2025] is another possible direction, in which a zig-zag shaped path in complex time includes classical evolution and tunneling in one common solution.

There is progress along all those lines, especially the second one. As shown in [2] turning states at different collision energies seem to be all sphalerons with the rescaled size. Also, the cross section look like a resonance, with a resemblance of Breit-Wigner peak.

As the calculation of the cross section from first principles is still not available, one has to use some phenomenological model. In [i $[10$ in] we estimated an upper limit for the number of sphaleron-type clusters produced from excited instantons in heavy ion collisions, ignoring shadowing and similar effects. For central $\mathrm{AuAu}$ and the value for the effective quarkquark-cluster cross section fitted to hadron-hadron data (see subsection above) we got about $\approx 400$ clusters. With 3 gluons plus 6 quarks per cluster, it will lead to 3600 partons, or the entropy comparable to the total one produced. Presumably the realistic number is a factor 2-3 lower; but in any case it is not a small effect.

\section{JET QUENCHING}

\subsection{Radiation in Various settings}

The basic new idea (which we propose to explain why jet quenching is so strong) is that during the first $\mathrm{fm} / \mathrm{c}$ the glue is a strong coherent field rather than a set of incoherent partons. We will below refer to this type of radiation as synchrotron-like. Strong external 
field problem requires knowledge of the exact trajectories (classically) or exact propagators (quantum mechanically). In QED synchrotron radiation usually takes place within a mag-

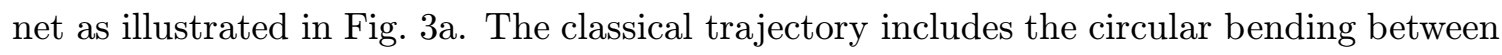
the incoming and outgoing straight lines, and the radiation is emitted tangent to the arc length. Photons move on straight lines since in QED they are not affected by the external magnetic field.

Another interesting case of classical synchrotron-like radiation is that of an ultrarelativistic rotating charge in a strong gravitational field such as the one encountered near the horizon of a black-hole [2] $\left.{ }^{2} \overline{\overline{6}}\right]$. In this case, both the charge and the photon are gravitationally deflected as illustrated in Fig. 3 . The result is a significant reduction of the radiation loss: the total radiation yield is reduced by a factor of $\gamma^{2}$ in comparison to the yield from standard synchrotron radiation for the same curvature. Also, the radiation length for each particular direction is actually the entire circle, not just an arc length of order $1 / \gamma$ as in the magnet.

In [i] $\overline{2} \overline{7}]$ we considered an ultrarelativistic charge in QCD (parton, quark, gluon) going through a constant chromomagnetic field as illustrated in Fig. tial charge and the ensuing radiation are both strongly affected by the chromomagnetic field. Moreover, the radiated gluons carry different charges and therefore move in different directions: some gluons however may be uncharged in respect to the field due to commuting color charges and behave as photons. The QCD chromomagnetic field resolves both momentum and color thereby acting as a double (squared) Newtonian prism.

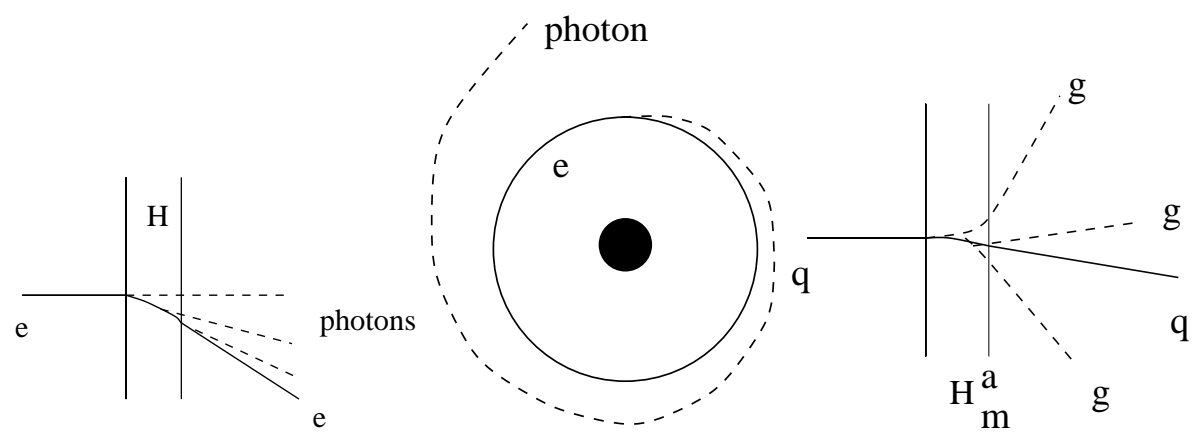

Figure 3: Schematic representation of synchrotron-like radiation in three cases: (a) in a magnetic field; (b) in a gravitational field (rotating charge around a black hole); (c) in a chromomagnetic field.

In [2] $\overline{7}]$ we estimated the QCD synchrotron radiation in a constant and Abelian-like chromomagnetic field. We derived the exact classical and first quantum correction in the regime $\omega / E<1$, and provided an approximate expression for all frequencies $\omega$. The problem of quantum synchrotron radiation in QED was addressed in a fundamental way by Schwinger [2] $\overline{8}]$ using the mass operator formalism, and we extended his approach to the quantum synchrotron radiation in QCD.

For simplicity, we consider QCD synchrotron radiation in a constant and homogeneous chromomagnetic field $G_{\mu \nu}^{a}(x)=\delta^{a 8} G_{\mu \nu}$ where the abelian field strength corresponds to a constant magnetic field in the 3-direction, $G_{12}=-G_{21}=H$. With our choice of the 
chromomagnetic background along the 8th color direction, the quarks and gluons can be diagonalized. There is difference for $A=4,5,6,7$ and $g_{A}=0$ for $A=1,2,3,8$ : the second case is basically QED-like. Quantum synchrotron radiation will be sought for quarks and gluons interacting to all orders in $H$ but to leading order in $\alpha=g^{2} / 4 \pi$ between the quantized fields. We obtain the chromomagnetic synchrotron emission by a scalar quark in the classical limit

$$
\begin{aligned}
\mathbf{P}_{a a}(\omega)= & -\frac{\alpha}{\pi}\left(T^{A}\right)_{a b}\left(T^{A}\right)_{b a} \omega \operatorname{Im} \int_{0-i 0}^{\infty-i 0} \frac{d \tau}{\tau} \frac{e^{-i\left(E \omega_{A}\right)^{2} \tau^{3} /(24 \omega)}}{\cos \left(E \omega_{A} \tau /(2 \omega)\right.} \\
& \times\left(\frac{m^{2}}{E^{2}}+\frac{1}{2} \omega_{b}^{2} \tau^{2}\right) e^{-i \omega\left(\frac{m^{2} \tau}{2 E^{2}}+\frac{\omega_{b}^{2} \tau^{3}}{24}\right)}
\end{aligned}
$$

where in $(\overline{1}, \overline{1} \cdot \overline{1})$ the $H=0$ subtraction is not explicitly shown but implied. The quark synchrotron and gluon rescaled frequencies are $\omega_{a}=e_{a} H / E$ and $\omega_{A}=g_{A} H / E$ respectively.

Before we discuss results, let us comment the integrand of $(5.51)$. The last exponent is due to charge curving, and is the same as in QED. It provides a rapidly oscillating phase at large $\omega$ and a corresponding cutoff. The first exponent in the integrand of $(\overline{5} . \overline{1})$ is new. It stems from the gluon rotation in the chromomagnetic field.

\subsection{Applications to heavy ion collisions}

We cannot go into history of jet quenching here, or compare our results with others. Using

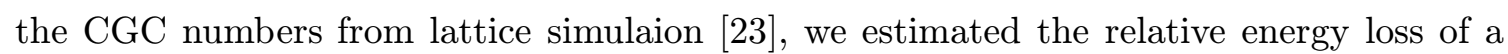
quark by synchrotron radiation in a time $\tau_{\mathrm{CGC}} \sim 1 / 2 \mathrm{fm} / \mathrm{c}$ to be

$$
\frac{\Delta E_{\mathrm{CGC}}}{E} \approx 0.3\left(\frac{H}{1 \mathrm{GeV}^{2}}\right)^{2 / 3}\left(\frac{\Delta \tau_{\mathrm{CGC}}}{0.5 f m}\right)\left(\frac{1 \mathrm{GeV}}{E}\right)^{1 / 3}
$$

The gluon loss is about twice the quark loss.

Now we proceed to topological clusters. We recall that upon release in Minkowski space, the sphaleron state explodes into thin shell: so field inhomogeneity provides another enhancement factor as compared to CGC, on top of coherence enhancement. At large time $t \gg \rho$ the corresponding gauge field is purely transverse, with equal chromoelectric and chromomagnetic fields and $\sqrt{H} \sim\left(\frac{2 M_{S}}{\rho^{3}}\right)^{1 / 4} \sim 1 \mathrm{GeV}$.. The optimal time is $2-3 \rho \sim$ $.7-1 \mathrm{fm} / \mathrm{c}$ when shells form a foam-like structure. Our estimated quark energy loss is

$$
\frac{\Delta E}{E} \sim 0.21\left(\frac{H}{0.2 \mathrm{GeV}^{2}}\right)^{2 / 3}\left(\frac{1 \mathrm{GeV}}{E}\right)^{1 / 3} .
$$

The gluon loss scales with the pertinent color Casimir and is again about twice larger. Both CGC and foam-like stage of cluster explosion provides significant jet quenching, comparable to what is needed to explain jet quenching by roughly one order of magnitude. It is also important that the radiated glue is emitted at large angles and cannot be recovered experimentally: this is in contrast to bremmstrahlung due to multiple small angle scattering, when radiation is emitted at small angles. We also comment that it is more important to evaluate the escape probability with no or little radiation, rather than the average loss: we hope this mechanism is very effective in this respect as well. 


\section{References}

[1] D. M. Ostrovsky, G. W. Carter and E. V. Shuryak, hep-ph/0204224.

[2] R. A. Janik, E. Shuryak and I. Zahed, "Prompt multi-gluon production in high energy collisions from singular Yang-Mills solutions," hep-ph/0206005.

[3] T. Schafer and E. V. Shuryak, Rev. Mod. Phys. 70, 323 (1998) [hep-ph/9610451].

[4] A.Ringwald, Nucl.Phys. B330 (1990) 1, O.Espinosa, Nucl.Phys. B343 (1990) 310; V.V. Khoze, A. Ringwald, Phys.Lett. B259:106-112, 1991 V.I.Zakharov, Nucl.Phys. B353 (1991) 683: M.Maggiore and M.Shifman, Phys.Rev.D46:3550-3564,1992 D. Diakonov and V. Petrov, Phys. Rev. D 50, 266 (1994) [hep-ph/9307356].

[5] E. Shuryak and I. Zahed, "Prompt quark production by exploding sphalerons," hep-ph/0206022.

[6] E. Shuryak, hep-ph/0205031.

[7] E. V. Shuryak, Nucl. Phys. B 203, 93 (1982).

[8] J. Zadrozny, Phys. Lett. B 284, 88 (1992). M. Hellmund and J. Kripfganz, Nucl. Phys. B 373, 749 (1992).

[9] D. E. Kharzeev, Y. V. Kovchegov and E. Levin, Nucl. Phys. A 690, 621 (2001) [hep-ph/0007182]. M. A. Nowak, E. V. Shuryak and I. Zahed, Phys. Rev. D 64, 034008 (2001) [hep-ph/0012232].

[10] G. W. Carter, D. M. Ostrovsky and E. V. Shuryak, Phys. Rev. D 65, 074034 (2002) [hep-ph/0112036].

[11] A.Ringwald, the talk at this conference.

[12] A.Brandt et al (UA8), hep-ex/0205037.

[13] B. Buschbeck, H. C. Eggers and P. Lipa, Phys. Lett. B 481, 187 (2000) [arXiv:hep-ex/0003029].

[14] D. Teaney, J. Lauret and E. V. Shuryak, Phys. Rev. Lett. 86,ace-str 4783 (2001) [arXiv:nucl-th/0011058], nucl-th/0110037. P. F. Kolb, P. Huovinen, U. W. Heinz and H. Heiselberg, Phys. Lett. B 500, 232 (2001) [hep-ph/0012137]. P. Huovinen, P. F. Kolb, U. W. Heinz, P. V. Ruuskanen and S. A. Voloshin, Phys. Lett. B 503, 58 (2001) [hep-ph/0101136].

[15] E. V. Shuryak, nucl-th/0112042

[16] E. V. Shuryak, Phys. Lett. B 78, 150 (1978) Sov. J. Nucl. Phys. 28, 408.1978 YAFIA,28,796 (1978). Phys. Rept. 61, 71 (1980).

[17] K. Geiger and B. Muller, Nucl. Phys. A 544, 467C (1992).

[18] R. Baier, A. H. Mueller, D. Schiff and D. T. Son, Phys. Lett. B 502, 51 (2001) [hep-ph/0009237]. hep-ph/0204211.

[19] L. Xiong and E. Shuryak, Nucl. Phys. A590, 589C (1995).

[20] D. Molnar and M. Gyulassy, Nucl. Phys. A 697, 495 (2002) [nucl-th/0104073].

[21] G. Policastro, D. T. Son and A. O. Starinets, Phys. Rev. Lett. 87, 081601 (2001) [hep-th/0104066]. 
[22] L. D. McLerran and R. Venugopalan, Phys. Rev. D 49, 2233 (1994) [hep-ph/9309289].

[23] A. Krasnitz, Y. Nara and R. Venugopalan, Phys. Rev. Lett. 87, 192302 (2001) [hep-ph/0108092].

[24] P. Levai and U. W. Heinz, Phys. Rev. C 57, 1879 (1998) [hep-ph/9710463].

[25] V. A. Rubakov and D. T. Son, Nucl. Phys. B 424, 55 (1994) [hep-ph/9401257].

[26] I.B.Khriplovich and E.V.Shuryak, Zh.Exp.Teor.Fiz.65 (1973) 2137; Sov.Phys.JETP, 38 (1974) 1067,

[27] E.V.Shuryak and I.Zahed, Cyclotron-like radiation in QCD,hep-ph/0207163.

[28] J.Schwinger, Phys.Rev.D 7 (1973) 1696 